\title{
Learning the optimal scale for GWAS through hierarchical SNP aggregation
}

\author{
Florent Guinot ${ }^{1,2^{*}}$ (D), Marie Szafranski ${ }^{1}$, Christophe Ambroise ${ }^{1,3}$ and Franck Samson ${ }^{1}$
}

\begin{abstract}
Background: Genome-Wide Association Studies (GWAS) seek to identify causal genomic variants associated with rare human diseases. The classical statistical approach for detecting these variants is based on univariate hypothesis testing, with healthy individuals being tested against affected individuals at each locus. Given that an individual's genotype is characterized by up to one million SNPs, this approach lacks precision, since it may yield a large number of false positives that can lead to erroneous conclusions about genetic associations with the disease. One way to improve the detection of true genetic associations is to reduce the number of hypotheses to be tested by grouping SNPS.

Results: We propose a dimension-reduction approach which can be applied in the context of GWAS by making use of the haplotype structure of the human genome. We compare our method with standard univariate and group-based approaches on both synthetic and real GWAS data.

Conclusion: We show that reducing the dimension of the predictor matrix by aggregating SNPs gives a greater precision in the detection of associations between the phenotype and genomic regions.
\end{abstract}

Keywords: Genome-wide association study, Statistical genetics, Variable selection, Hierarchical clustering

\section{Background \\ Context}

Recent breakthroughs in microarray technology have meant that hundreds of thousands of single nucleotide polymorphisms (SNPs) can now be densely genotyped at moderate cost. As a result it has become possible to characterize the genome of an individual with up to a million genetic markers. These rapid advances in DNA sequencing technologies have also made it possible to carry out exome and whole-genome sequencing studies of complex diseases. In this context, Genome-Wide Association Studies (GWAS) have been widely used to identify causal genomic variants implied in the expression of different human diseases (rare, Mendelian, or multifactorial diseases).

From a statistical point of view, looking for these variants can be supported by hypothesis testing. The standard approach in GWAS is based on univariate regression
*Correspondence: florent.guinot@genopole.cnrs.fr

'UMR 8071 LaMME - UEVE, CNRS, ENSIIE, USC INRA, 23 bd de France, 91000

Evry, France

${ }^{2}$ BIOptimize, 51000 Reims, France

Full list of author information is available at the end of the article (logistic regression in case-control studies), with affected individuals being tested against healthy individuals at one or more loci. Classical testing schemes are subject to false positives (that is to say SNPs that are falsely identified as significant variables). One way around this problem is to apply a correction for the False Discovery Rate $[1,2]$. Unfortunately, this increases the risk of missing true associations that have only a small effect on the phenotype, which is usually the case in GWAS. [3] suggested that standard approaches such as multiple hypothesis testing may not be appropriate for the detection of small effects from multiple SNPs. In such cases a significant part of the heritability can be missing and GWAS fails to detect all possible genetic variants associated with a disease.

Furthermore, this kind of standard approach faces other limitations:

1. It does not directly account for correlations among the predictors, whereas these correlations can be very strong as a result of linkage disequilibrium (LD). SNPs can be correlated even where they are not physically linked, because of population structure or epistasis (gene by gene interactions).

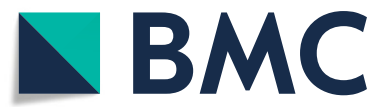

(c) The Author(s). 2018 Open Access This article is distributed under the terms of the Creative Commons Attribution 4.0 International License (http://creativecommons.org/licenses/by/4.0/), which permits unrestricted use, distribution, and reproduction in any medium, provided you give appropriate credit to the original author(s) and the source, provide a link to the Creative Commons license, and indicate if changes were made. The Creative Commons Public Domain Dedication waiver (http://creativecommons.org/publicdomain/zero/1.0/) applies to the data made available in this article, unless otherwise stated. 
2. It does not account for epistasis, i.e. causal effects that are only observed when certain combinations of mutations are present in the genome.

3. It does not directly provide predictive models for estimating the genetic risk of the disease.

4. It focuses on identifying common markers with allele-frequency (MAF) above 5\%, although it is likely that analyzing low-frequency $(0.5 \%<\mathrm{MAF}<5 \%)$ and rare $(\mathrm{MAF}<0.5 \%)$ variants would be able to explain additional disease risks or trait variability [4].

Uncovering some of the missing heritability can sometimes be achieved by taking into account correlations among variables, interaction with the environment, and epistasis, but this is rarely feasible in the context of GWAS because of the multiple testing burden and the high computational cost of such analyses [5]. In the context of rare-variant association analysis, a number of region- or gene-based multimarker tests have been proposed as burden tests [6], variance-component tests [7] or combined burden and variance component tests [8]. Instead of testing each variant individually, these methods evaluate the cumulative effects of multiple genetic variants in a gene or a region, increasing power when multiple variants in the group are associated with a given disease or trait.

Furthermore, regarding limitation (4), analyzing rare variants is more complex than analyzing more common variants and a large sample size is needed to observe a rare variant with a high probability.

\section{Group and aggregation based methods for common variants}

Although classical GWAS have limitations that prevent a full understanding of the heritability of genetic and/or multifactorial diseases, there are nevertheless ways of overcoming these limitations to some degree. For instance, it is possible to take into account the structure of the data in the hypothesis testing procedure. As an illustration, [9] proposed a hierarchical testing approach which considers the influence of clusters of highly correlated variables rather than individual variables. The statistical power of this method to detect relevant variables at single SNPs level was comparable to that of the Bonferroni-Holm procedure [10], but the detection rate was much higher for small clusters, and it increased further at coarser levels of resolution.

Group-based methods require an appropriate group definition. In GWAS, the usual approach is to group SNPs which are included in the same gene but this limits the analysis to coding regions. It is well known that the human genome is structured into haplotype blocks, i.e. sizable regions over which there is little evidence for historical recombination and within which only a few common haplotypes may be observed [11]. The boundaries of blocks and the specific haplotypes that they contain are highly correlated across populations [12]. With this property of the human genome in mind, [13] developed a method for detecting haplotype-disease associations in genomewide studies, based on sliding windows of adjacent SNPs, along with a Monte Carlo procedure to adjust for multiple testing.

In [14], the authors proposed to group SNPs into sets on the basis of their proximity to genomic features such as genes or haplotype blocks and then to identify the joint effect of each set via a logistic kernel-machine-based test. This approach lays the foundation for the Sequence Kernel Association Test method [7].

In the broad family of linear models, [15] introduced a likelihood ratio-based set test that accounts for confounding structure. The model is based on the linear mixed model and uses two random effects, one to capture the set association signal and one to capture confounders. They demonstrate a control of type I error as well as an improved power over more traditionally used score test. Other methods focus on multiple linear regression either by taking into account the linkage disequilibrium within the genes to improve power [16] or by clustering variants with weak association around known loci to increase the percentage of variance explained in complex traits [17].

Finally, other approaches will focus on the aggregation of summary statistics of single SNPs within a same gene with for instance the data-driven aggregation of summary statistics described in [18] or the procedures of $p$-value combination in [19]. In the cited articles, the methods are used on SNPs located in coding region (or extended intronic region in [19]) but can be extended to any set of SNPs as long as we pre-specified a set of variants within a region. However the power for each test remains dependent of the true disease model. Furthermore, this kind of approaches may also lose statistical power in comparison to single-variant-based tests when only a very small number of the variants in a gene are associated with the trait, or when many variants have no effect or causal variants are low-frequency variants [4].

\section{Organisation of the paper}

The present paper proposes a block-wise approach for GWAS analysis which leverages the LD structure among the genomic variants to reduce the number of hypotheses testing. We aggregate the SNPs into different clusters according to their LD levels and use a supervised learning approach to identify the clusters of SNPs related to a case-control phenotype. Our algorithm provides a group structure for the variables, enabling us to define a function 
that aggregates these clusters into new variables to be used in the GWAS machinery. The advantage of this method is that aggregating clusters of several SNPs into a single variable reduces the dimension of the data without loss of information, since we are grouping variables that are highly correlated (in strong LD).

We compare our method in different scenarios with the baseline approach, i.e. univariate hypothesis testing [20] and with a state-of-the-art method, the logistic kernel machine method developed by [14] on both synthetic and real datasets from the Wellcome Trust Case Control Consortium [21] and on ankylosing spondylitis data [22].

\section{Method}

In this section we describe a new method for performing GWAS using a four-step method that combines unsupervised and supervised learning techniques. This method improves the detection power of genomic regions implied in a disease while maintaining a good interpretability. This method consists in:

1. Performing a spatially constrained Hierarchical Agglomerative Clustering (constrained-HAC) of the SNPs matrix X using the algorithm developed by [23].

2. Applying a function to reduce the dimension of $\mathrm{X}$ using the group definition from the constrained-HAC. This step is described and illustrated in Fig. 1.

3. Estimating the optimal number of groups using a supervised learning approach to find the best cut into the hierarchical tree (cut level algorithm). This algorithm combines Steps 1 and 2 into an iterative process.

4. Applying the function defined in Step 2 to each group identified in Step 3 to construct a new covariate matrix and perform multiple hypothesis testing on each new covariate to find significant associations with a disease phenotype $y$.

\section{Step 1. Constrained-HAC}

In GWAS, the covariates are ordinal and correspond to SNP genotypes such that $X_{i j} \in\{0,1,2\}$ corresponds to the number of minor alleles at locus $j \in[1, \ldots, J]$ for observation $i \in[1, \ldots, N]$.

To take into account the structure of the genome in haplotype blocks, we group the predictors (SNPs) according to their LD in order to create a new predictor matrix which reflects the structure of the genome. We first use the algorithm developed by [23], which clusters SNPs into adjacent blocks. The clustering method is a spatially constrained hierarchical clustering based on Ward's incremental sum-of-squares algorithm [24], in which the measure of dissimilarity is not based on the Euclidean distance but rather on the linkage disequilibrium between two SNPs: $1-r^{2}\left(j, j^{\prime}\right)$. The algorithm also makes use of the fact that the LD matrix can be modeled as block-diagonal by allowing only groups of variables that are adjacent on the genome to be merged, which significantly reduces the computation cost. This algorithm is available via the $R$ package called adjclust on https://cran.r-project.org/ web/packages/adjclust.
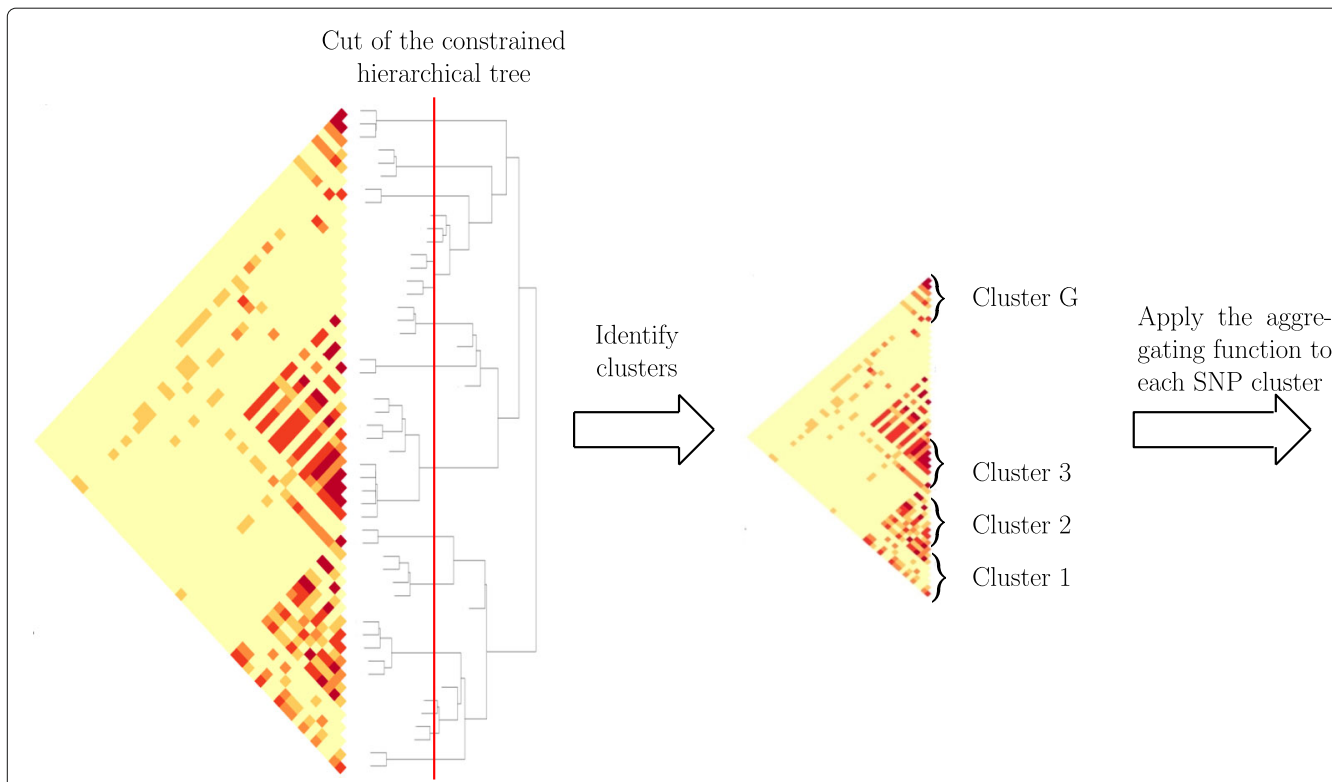

New predictor matrix

D with $G$ covariates

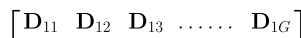

$\left.\begin{array}{lllll}\mathbf{D}_{21} & \mathbf{D}_{22} & \mathbf{D}_{23} & \ldots \ldots & \mathbf{D}_{2 G} \\ \mathbf{D}_{31} & \mathbf{D}_{32} & \mathbf{D}_{33} & \ldots & \mathbf{D}_{3 G}\end{array}\right]$

$\begin{array}{llllll}\mathbf{D}_{41} & \mathbf{D}_{42} & \mathbf{D}_{43} & \ldots \ldots & \mathbf{D}_{4 G}\end{array}$

$\begin{array}{cccccc}\mathbf{D}_{41} & \mathbf{D}_{42} & \mathbf{D}_{43} & \ldots \ldots & \mathbf{D}_{4 G} \\ \vdots & \vdots & \vdots & \ldots \ldots & \vdots \\ \vdots & \vdots & \vdots & \ldots \ldots & \vdots \\ \mathbf{D}_{N 1} & \mathbf{D}_{N 2} & \mathbf{D}_{N 3} & \ldots \ldots & \mathbf{D}_{N G}\end{array}$

Fig. 1 Schematic view of Step 2 of the algorithm to calculate the matrix of predictors D 


\section{Step 2. Dimension reduction function}

One way of addressing issues related to high-dimensional statistics (and in particular the multiple testing burden that we mentioned above) is to reduce the dimensionality of the predictor matrix $\mathrm{X} \in \mathbb{R}^{N \times J}$ by creating a reduced matrix D with new covariates that nevertheless remain representative of the initial matrix. This means reducing the number of predictors $J$ to $G \ll J$, with row $\mathrm{D}_{i}$. the $G$-dimensional vector of new predictors for observation $i$. In this study we use a blockwise approach to construct a matrix of new uncorrelated predictors $\mathrm{D} \in \mathbb{R}^{N \times G}$, with $G$ the number of groups in linkage disequilibrium identified via the constrained agglomerative hierarchical clustering described in Step 1.

While classical methods use the initial set of covariates to predict a phenotype, we propose combining a clustering model with a dimension reduction approach in order to predict $y$. For each group identified with the constrained-HAC, we apply a function to obtain a single variable defined as the number of minor alleles present in the group. For each observation $i$ and in each cluster $g \in[1, \ldots, G]$, the variable is defined as:

$$
\mathrm{D}_{i g}=\sum_{j \in g} \mathrm{X}_{i j}
$$

In order that the values for the different groups are comparable, we eliminate the effect of group size by centering and scaling the matrix $\mathrm{D}$ to unit variance. In the remainder of the paper we will refer to the covariates in D as aggregated-SNP variables.

\section{Step 3. Optimal number of groups estimation}

Estimating the optimal number of groups to select, i.e. the level at which the hierarchical clustering tree should be cut, is a fundamental matter which impacts the relevance of the association analysis. It is known that the human genome is structured into haplotype blocks with little or no within-block recombination [12], but it is not easy to determine how these blocks are allocated throughout the genome for a given set of SNPs.

In the literature, in an unsupervised learning context, a number of models have been proposed for determining this optimal number of groups [25-28]. These methods are all based on the measure of within-group dispersion $W_{G}$ with $G \in[1, \ldots, P]$. Since GWAS consist in evaluating the likelihood of the disease from genetic markers, we propose using the phenotype $y$ as a way of determining the optimal number of clusters.

We propose here a supervised validation set approach to find this optimum. Since this algorithm aims to identify phenotype-related SNPs clusters, it is necessary to split the dataset into two subsets to avoid an inflation of type I errors in the testing procedure. One subset, [ $\left.Y_{s u b 1}, \mathrm{X}_{s u b 1}\right]$, is used to choose the optimal cut and the second one, $\left[Y_{\text {sub } 2}, \mathrm{X}_{\text {sub } 2}\right]$, to perform the hypothesis testing in Step 4.

First we apply the constrained-HAC on a subset $\mathrm{X}_{\text {subl } 1}^{\text {train }} \subset$ $\mathrm{X}_{s u b 1}$, and for a given level of the hierarchy we apply the dimension reduction function defined above (Step 2) to each of the $G$ clusters to construct the matrix $\mathrm{D}_{s u b 1}^{\text {train }}$. We then fit a ridge regression model to estimate the coefficients of the predictors in $\mathrm{D}_{\text {sub } 1}^{\text {train }}$. Ridge regression is a penalized model which shrinks the estimated coefficients towards zero and is known to have a good stability in comparison to other penalized-regression models such as lasso regression [29]. Moreover, a link can be established between the ridge regression model and the mixed linear model used in the estimation of the heritability in a high-dimensional setting [30]. Once the coefficients are estimated, we predict the phenotypic values on the test set using the matrix $\mathrm{D}_{\text {sub } 1}^{\text {test }}$ and calculate either the mean test set error when the phenotype is quantitative or the Area Under the ROC curve (AUC-ROC) when it is binary. The procedure, summarized in Algorithm 1, is then repeated for different levels in the hierarchy and the optimal cut level in the tree is defined as the level which maximizes the prediction accuracy criterion.

\section{Step 4. Multiple testing on aggregated-SNP variables}

Once the optimal number of groups has been determined, we apply the function (1) to each selected group and construct the matrix of aggregated-SNP. Here we use a standard Single Marker Analysis (SMA) to find associations with the phenotype, but instead of calculating

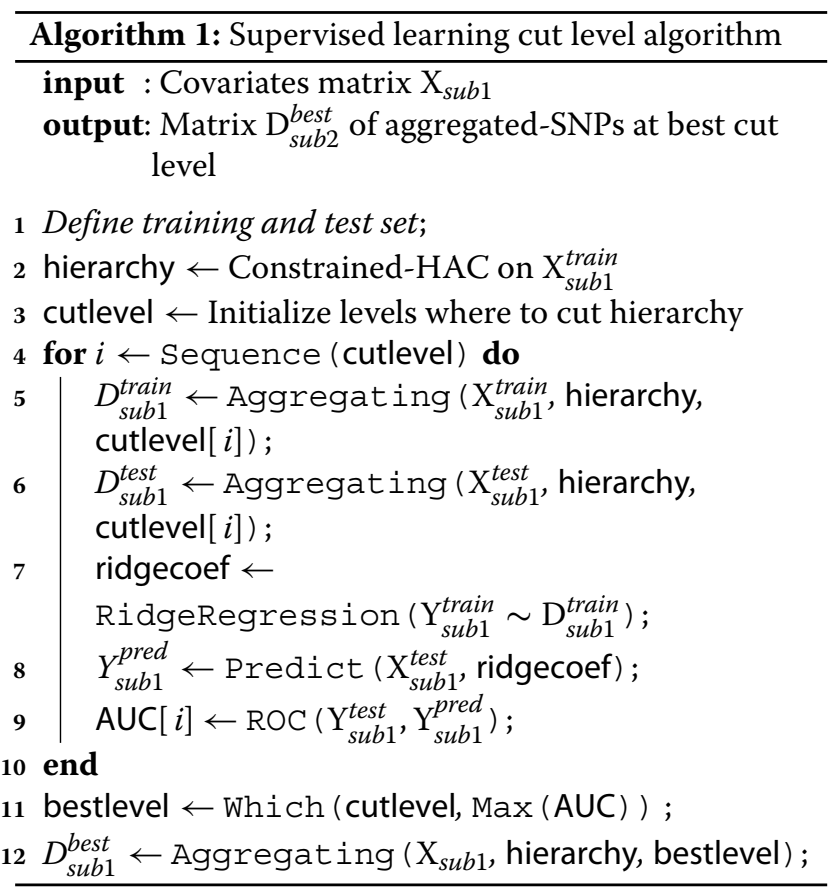


$p$-value for each SNPs in $\mathrm{X}_{\text {sub2 } 2}$, we calculate $p$-value for each aggregated-SNP variables in $\mathrm{D}_{s u b 2}$.

As in standard SMA, a univariate generalized linear model [31] is fitted for each variable $\mathrm{D}_{j}: f\left(\mu_{i}\right)=\mathrm{D}_{i j} \beta$, where $\mu_{i} \equiv \mathbb{E}\left(\mathrm{Y}_{i} \mid \mathrm{D}_{i}\right)$ $\left(\mathrm{Y}_{\mathrm{i}} \sim\right.$ some exponential family distribution $), f$ is a smooth monotonic 'link function, $\mathrm{D}_{i j}$ is the $i^{\text {th }}$ row of the model matrix $\mathrm{D}_{. j}$ of aggregate-SNP and $\beta$ is a vector of 2 unknown coefficients with $\beta_{0}$ for the intercept and $\beta_{1}$ for the predictor $j$. Where the response variable is a binary trait (i.e. case-control phenotype), we use the logit function as the 'link function' $f$ and $Y_{i} \sim$ Bernoulli distribution. This model is known as the logistic regression model. Then, for each single-predictor model, we perform a Likelihood Ratio Test where we compare the intercept-only model against the singlepredictor model and get for each predictor a $p$-value using the $\tilde{\chi}^{2}$ distribution.

Given that a large number of covariates are being tested, we need to compute an appropriate significance threshold to control the family-wise error rate, $F W E R=\mathbb{P}(F P>1)$, with $F P$ being the False Positive, since keeping the threshold at the conventional value of $\alpha=0.05$ would yield numerous false positives. Several approaches, including the Bonferroni correction, have been proposed in the context of genetic studies for controlling the FWER [32]. An alternative approach, developed by [1], seeks to control the False Discovery Rate (FDR) which is the expectation of ratio between the number of false positives and the total positive outcomes: $F D R=\mathbb{E}\left(\frac{F P}{F P+T P}\right)$, with $T P$ being the True Positive. The Bonferroni correction reduces the significance level according to the number of tests carried out in the study. However, in the context of GWAS, where hundreds of thousands of tests have to be performed, the Bonferroni correction is too strong, and will often decrease the significance threshold to a level where almost nothing is significant. Controlling FDR is therefore preferable. It is an approach that is less stringent but nonetheless powerful. The method for controlling FDR does not directly set a significance threshold, but rather identifies the largest $p$-value that is substantially smaller than its expected value (by a factor of at least $1 / \phi$ where $\phi$ is the desired FDR level), given that all the tests follow $H_{0}$. The $p$-value thus identified and all smaller $p$-values are deemed to be significant.

\section{Numerical simulations}

The performance evaluation described below was designed to assess the ability of our method to retrieve causal SNPs or causal clusters of SNPs under different simulation scenarios. For each scenario, we use a matrix $\mathrm{X}_{\text {HAPGEN }}$ of SNPs generated by the HAPGEN2 software [33] with a sample size of 1000 individuals. This software allows to simulate an entire chromosome conditionally on a reference set of population haplotypes (from HapMap3) and an estimate of the fine-scale recombination rate across the region, so that the simulated data share similar patterns with the reference data. We generate the chromosome 1 (103 457 SNPs) using the haplotype structure of CEU population (Utah residents with Northern and Western European ancestry from the CEPH collection) as reference set. The HAPGEN2 software allows to generate a controls-only matrix of SNPs (no disease allele). We filtered this matrix according to the minor allele frequency to only keep SNPs with a MAF greater than 5\% thus reducing the size of $\mathrm{X}_{\text {HAPGEN }}$ to 60179 SNPs.

We generate a posteriori the phenotype using the logit model with a given set of causal SNPs or cluster of SNPs. The main difference between the different scenarios is to be found in the way that the case-control phenotype $y$ is simulated.

\section{Simulation of the case-control phenotype}

For all simulation scenarios, we simulated a case-control phenotype $y$ under a logistic regression model. The casecontrol phenotype is generated following a Bernoulli distribution function, following the conditional probability $\mathbb{P}\left(y_{i}=1 \mid \tilde{\mathrm{X}}_{i .}\right)$ with $\tilde{\mathrm{X}} \in \mathbb{R}^{n \times \ell}$ a matrix constructed by

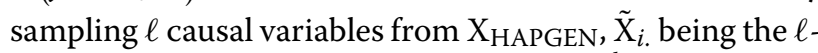
dimensional vector corresponding to the $i^{\text {th }}$ observation.

The conditional probability is calculated using the logit model:

$$
\mathbb{P}\left(y_{i}=1 \mid \tilde{\mathrm{X}}_{i .}\right)=\frac{\exp \left(\beta_{0}+\boldsymbol{\beta} \tilde{\mathrm{X}}_{i .}\right)}{1+\exp \left(\beta_{0}+\boldsymbol{\beta} \tilde{\mathrm{X}}_{i .}\right)},
$$

where $\boldsymbol{\beta}=\left[\beta_{1}, \ldots, \beta_{\ell}\right]$ is the vector of coefficients corresponding to the $\ell$ predictors $\left[\tilde{\mathrm{X}}_{.1}, \ldots, \tilde{\mathrm{X}}_{. \ell}\right]$ and $\beta_{0}$ is the intercept defined as $\ln \left(\frac{\pi}{(1-\pi)}\right)$, with $\pi$ the true prevalence of the disease in the population. The predictors are centered to have zero-mean before generating the vector of probability.

One way to have an association between the response and the predictors strong enough to be detected is to set large $\boldsymbol{\beta}$ coefficients on the predictors. Indeed there is a direct relationship between the odd ratio $(O R)$ of a covariate $\tilde{\mathrm{X}}_{i \text {. }}$ and its corresponding coefficients $\boldsymbol{\beta}_{i}$ in the logistic regression model [34] given by $O R_{i}=e^{\left(\boldsymbol{\beta}_{i}\right)}$. In our simulations, the difficulty of the problem, i.e. the power to detect an association, is linked to the number of causal predictors used to generate $y$ and the $O R$ set to each predictors.

To simulate different scenarios we considered the following parameters:

1. Nature of the causal predictors:

- Clusters of SNP: For each replicates, $\ell=1,2,3$ genomic regions have been identified to be 
causal. These regions have been chosen among the matrix $\mathrm{X}_{\text {HAPGEN }}$ to have different levels of LD among the SNPs that compose them. The average correlation coefficient among the SNPs in these regions varies from $r^{2}=0.6$ to $r^{2}=0.85$ and the size of the region varies from 20 SNPs to 60 SNPs. Once identified, the causal regions were aggregated using the function described in Step 2 to construct a matrix $\tilde{X}$ of aggregated-SNPs predictors used to generate the case-control phenotype. Under this scenario, all the SNPs within the region in LD can be considered as causal but it is the aggregated variables that is used to generate the phenotype. We will refer to it as the SNPclus scenario.

- Single SNPs. In this scenario the phenotype was simulated by directly using sampled SNPs from the same causal regions identified in the SNPclus scenario. For each replicates, we chose 10 individuals SNPs among each of these regions to construct a matrix $\tilde{\mathrm{X}}$ with $\ell=10,20,30$ single SNPs predictors, depending on the number of causal regions. This matrix is then used to generate the case-control phenotype. The chosen SNPs have a MAF varying from $10 \%$ to $30 \%$. We will refer to this scenario as the singleSNP scenario.

2. Number of causal predictors $\ell$ and number of replicates:

We performed 5 replicates for each combination $\ell \times$ number of scenarios and we evaluate the average performance over these 5 replicates. For each scenario we considered from 1 to 3 causal genomic regions, thus, for SNPclus scenario, we used up to 3 causal predictors, and for the singleSNP scenario, up to $10 \times 3=30$ causal predictors to generate the case-control phenotype.

3. Odd ratio ( $\beta$ coefficients) of the causal predictors: For the SNPclus scenario we chose an equal OR of 2.7 for each causal aggregated predictors, corresponding to a $\boldsymbol{\beta}$ coefficient equal to 1 . For the singleSNP scenario we chose an equal OR of 1.1 for each causal predictors, corresponding to a $\boldsymbol{\beta}$ coefficient equal to 0.1 . The rationale behind these coefficients arises from the hypothesis that the combined effect of several low-effect SNPs on the phenotype is stronger than the effects of each individual SNP.

As previously mentionned, we generated the phenotype using causal SNPs simulated with the HAPGEN2 software. However, as commercial genechips such as Affymetrix and Illumina arrays do not genotype the full sequence of the genome, some SNPs are thereby unmapped and the marker density is in general lower than the HapMap marker density. That is why we chose, in our numerical simulation, to generate the phenotype with causal variables chosen from the HAPGEN matrix and to assess the performance of the methods using only those SNPs which are mapped on a standard Affymetrix genechip (about 23823 mapped SNPs). By doing so, some causal SNPs are not mapped on the commercial SNP set and thus simulations are more similar to real genomewide analysis conditions.

\section{Results}

\section{Performance evaluation}

Competitors The objective of our method being to identify the optimal scale at which to perform association studies, we compared our proposal with several methods working at different genomic scales. The purpose is to assess the ability of each method to retrieve true causal genomic regions in the different simulation scenarios. For each scenario, four approaches have been considered:

- SKATtree, a SKAT model using our group definition,

- SKATnotree, a SKAT model using an alternative group definition produced by successive chunks of 20 SNPs,

- SMA, the classical Single Marker Analysis,

- SASA (Single Aggregated-SNP Analysis) a method close to SMA, where instead of testing the genotypephenotype association using each single SNP, we are testing it using aggregated-SNP variables.

The two above described group definitions for SKAT were considered to evaluate the impact of the group structure on the association findings.

The comparison with SMA allows to highlight the advantage of working at a group scale. We hypothesize that grouping low-effect SNPs should have a better statistical power than testing the main effects at single-SNP level.

For all methods, we compare the results using 2 types of multiple testing corrections : the methods of HolmBonferroni [10] and [1].

True and False Positive definitions. The problem of retrieving true causal associations can be represented as a binary decision problem where the compared methods are considered as classifiers. The decision made by a binary classifier can be summarized using four numbers: True Positives $(T P)$, False Positive $(F P)$, True Negatives $(T N)$ and False Negatives $(F N)$. We represent True Positive Rate $($ Recall or Power $=T P /(F N+T P))$ versus Precision $($ Precision $=T P /(F P+T P))$.

In this context, a True Positive corresponds to a true causal genomic region associated to significant $p$-value. 
The definition of what can be considered as the true causal genomic region may nevertheless be subject to some ambiguity. In GWAS, the presence of LD between SNPs often leads to consider the signal associated to multiple neighboring SNPs as indicating the existence of a single genomic locus with possible influence on the phenotype.

In our simulations, a causal genomic region is defined a priori as a causal predictor in the logit model. However, since the clusters of SNPs identified by our algorithm are not totally independent, some residual correlation may remain between clusters. This leads to question the notion of relevant variable when the variables are structured into strongly correlated groups. Should all the variables of the block be considered as explanatory, or should we define as only true positives the causal variables used to generate the phenotype?

In order to circumvent this issue, we chose to relax the definition of a False Positive joining the work of [35] and [36] where they propose to control the FDR in GWAS by considering significiant SNPs correlated to the true causal variables as true positives.

For the simulation of the phenotype, we hypothesize an underlying multivariate regression model, but test for univariate model as it is the usual practice, which leads to reconsider the definition of true positive. As in [36] we consider the set of true positive as the union of the causal true positive and the linked true positive, which are regions adjacent to the causal regions and correlated with them at a level of at least 0.5. Regarding the single-marker analysis approach, since it works at the single SNP level, we compare it with the others in the singleSNP scenario only.

\section{Results and discussions of the numerical simulations}

Area Under the ROC Curve. For each simulation, the cut level algorithm was applied. We recall that this algorithm calculates a prediction error on a test set for several levels in a constrained-HAC tree with a ridge regression model and chooses the level for which this error is the smallest. The AUC-ROC is plotted for the different levels, and the best cut level corresponds to the level for which AUC-ROC is the greatest. The results from the simulation scenario clusSNP and singleSNP described in "Simulation of the case-control phenotype" section are shown in Fig. 2. Our algorithm cuts the hierarchy either at a fairly high level (few large clusters) or at a low level (many small clusters), depending on the number of causal variables we used to generate the phenotype. The more the number of causal regions decreases, the higher the algorithm cuts in the hierarchical tree. In either case our algorithm is able to increase the predictive power by aggregating SNPs with the function (1).
We are thus able build a matrix of uncorrelated aggregated-SNP predictors that are representative of the initial SNP matrix and strongly linked to the phenotype.

Performance results for simulated data. As described in the performance evaluation section, we evaluate and compare the methods using two metrics, namely Recall or Power $=T P /(F N+T P)$ and Precision = $T P /(F P+T P)$.

Here the Precision metric is somewhat relaxed compared to its true definition since we adapted the definition of a True Positive and False Positive to the GWAS context. It is important to note that for all the methods, we compare the Benjamini-Hochberg method to control FDR with the Bonferroni correction to control FWER at a threshold of $5 \%$. However, since there are residual correlations between SNPs clusters and that the replication of numerous samples per combination of parameters is difficult in this realistic setting of simulations, the observed Type I error rate may be greater than $5 \%$. What we think is important to put forward to in these simulations is the ability of our algorithm to define groups of relevant clusters that will be detected on average with more precision and more power (SASA and SKAT tree) than using an arbitrary group definition (SKATnotree) or no definition of groups at all (SMA).

The results represented in Fig. 3 show that the methods using our algorithm for the cluster definition (SASA and SKATtree) have in average a better precision than the two other methods. The approach SASA, which combine our clustering algorithm and the aggregating function (1) to test the association of aggregated-SNPs with the phenotype, perform poorly in term of Recall but is far better in term of Precision compared to SMA and SKATnotree. These results suggest that it is better to combine our algorithm with the SKAT method than with the SASA method. We also note that applying the SKAT approach on an arbitrary group definition (SKATnotree) lead to a good recall but a very poor precision, showing the benefit of using our custom group definition in this context. Regarding the SMA approach in the singleSNP scenario, we can observe a loss in term of Recall compare to the SKAT tree and SKATnotree method suggesting that we can take benefit of grouping low effect SNPs to improve the power to detect causal genomic regions.

In GWAS, having a method with a good precision is as important, or even more important, than having a good recall. It is better to spot a few significant associations with a high certainty than to spot numerous significant associations but with only a low level of certainty for most of them. For this reason, we believe that our method represents an improvement in terms of precision without loss 


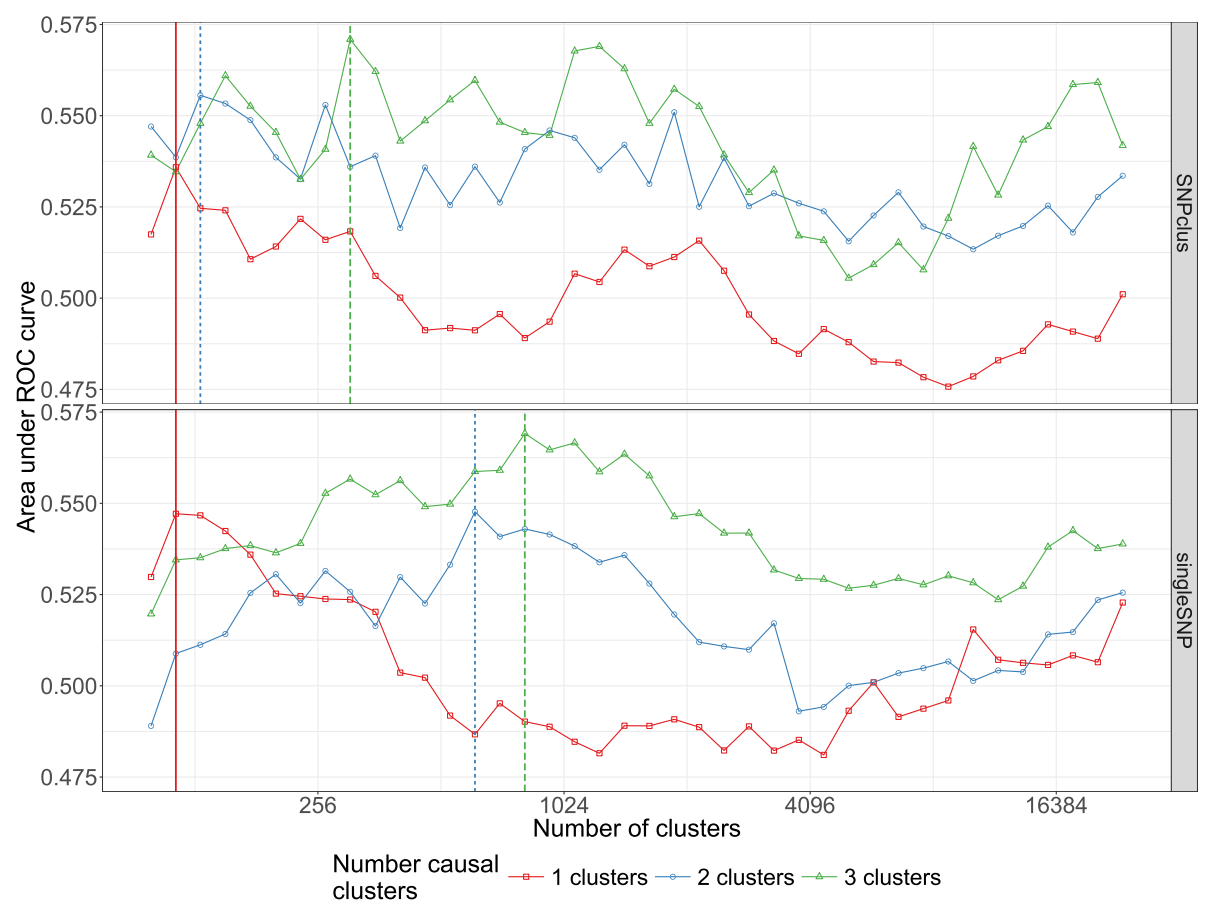

Fig. 2 Area under ROC curves according to the number of clusters in the clusSNP and singleSNP scenarios: the vertical lines indicate the number of aggregated-SNPs (clusters) obtained with Algorithm 1, i.e. the level where the prediction error is minimized (AUC-ROC at its maximum)

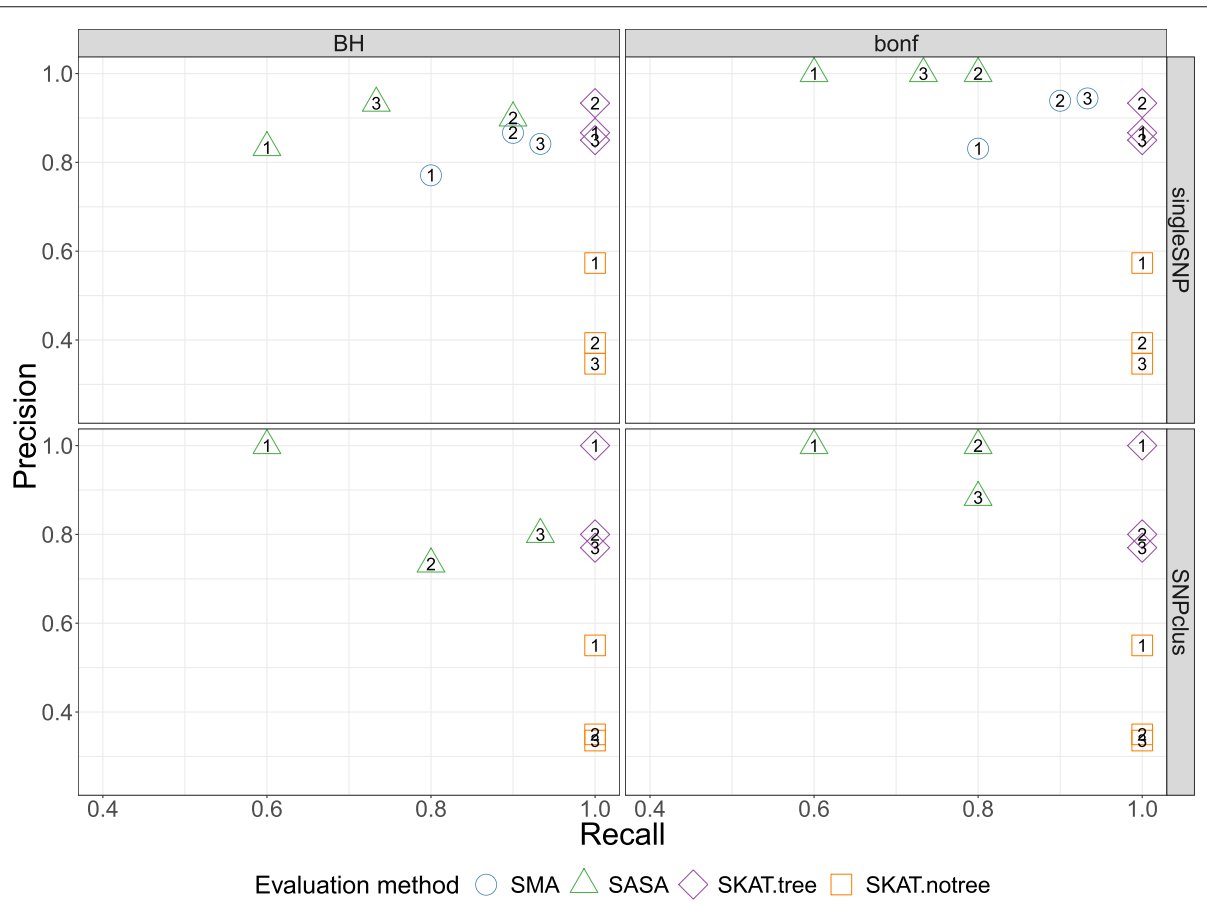

Fig. 3 Recall vs Precision for each method (shape and colors in plot). In rows are the simulation scenarios. In columns, we evaluate performance using Benjamini-Hochberg threshold (left) and bonferroni correction threshold (right). The second row illustrates the performance to retrieve the true causal genomic region under the SNPClus scenario, thus only group-based approaches are considered (SASA, SKAT.tree and SKAT.notree). The numbers inside the points correspond to the number of causal predictors and each point is the average value of 5 replicates 


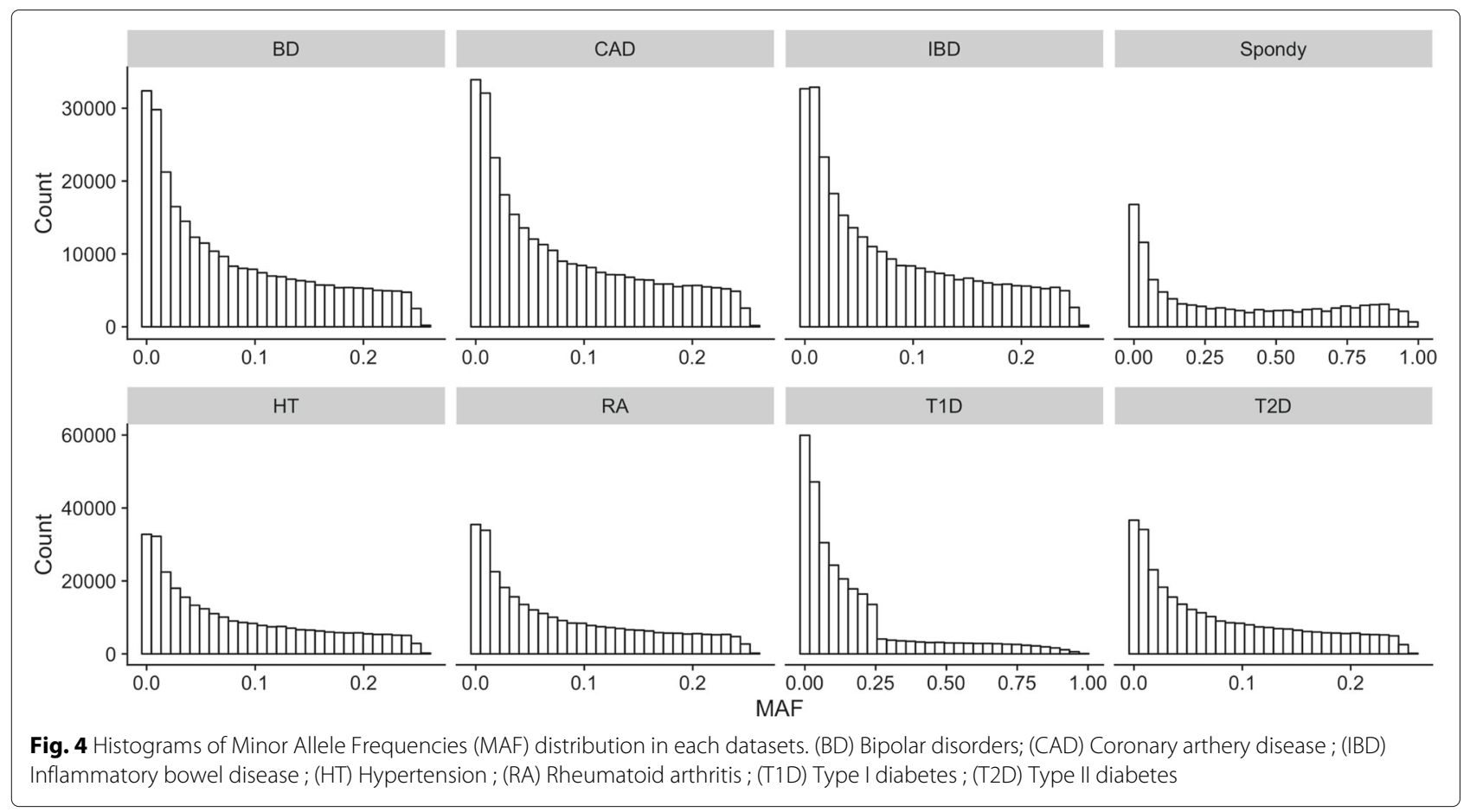

of power insofar as SKATtree seems able to detect significant genomic regions associated with the phenotype with a higher degree of certainty than standard approaches.

\section{Application in wellcome trust case control consortium(WTCCC) and Ankylosing Spondylitis (AS) studies}

To evaluate the performance of our method on real data, we performed GWAS analysis on datasets made available by [21]. The WTCCC data collection contains 17000 genotypes, composed of 3000 shared controls and 14000 cases representing 7 common diseases of major public health concern: inflammatory bowel disease (IBD), bipolar disorder (BD), coronary artery disease (CAD), hypertension (HT), rheumatoid arthritis (RA), and Type I (T1D) and Type II (T2D) diabetes. Individuals were genotyped with the Affymetrix GeneChip 500K Mapping Array Set and are represented by about 500,000 SNPs (before the application of quality control filters).

In parallel to the analysis of the WTTCC data, we decided to assess our method on another dataset from a different study. The ankylosing spondylitis (AS) dataset

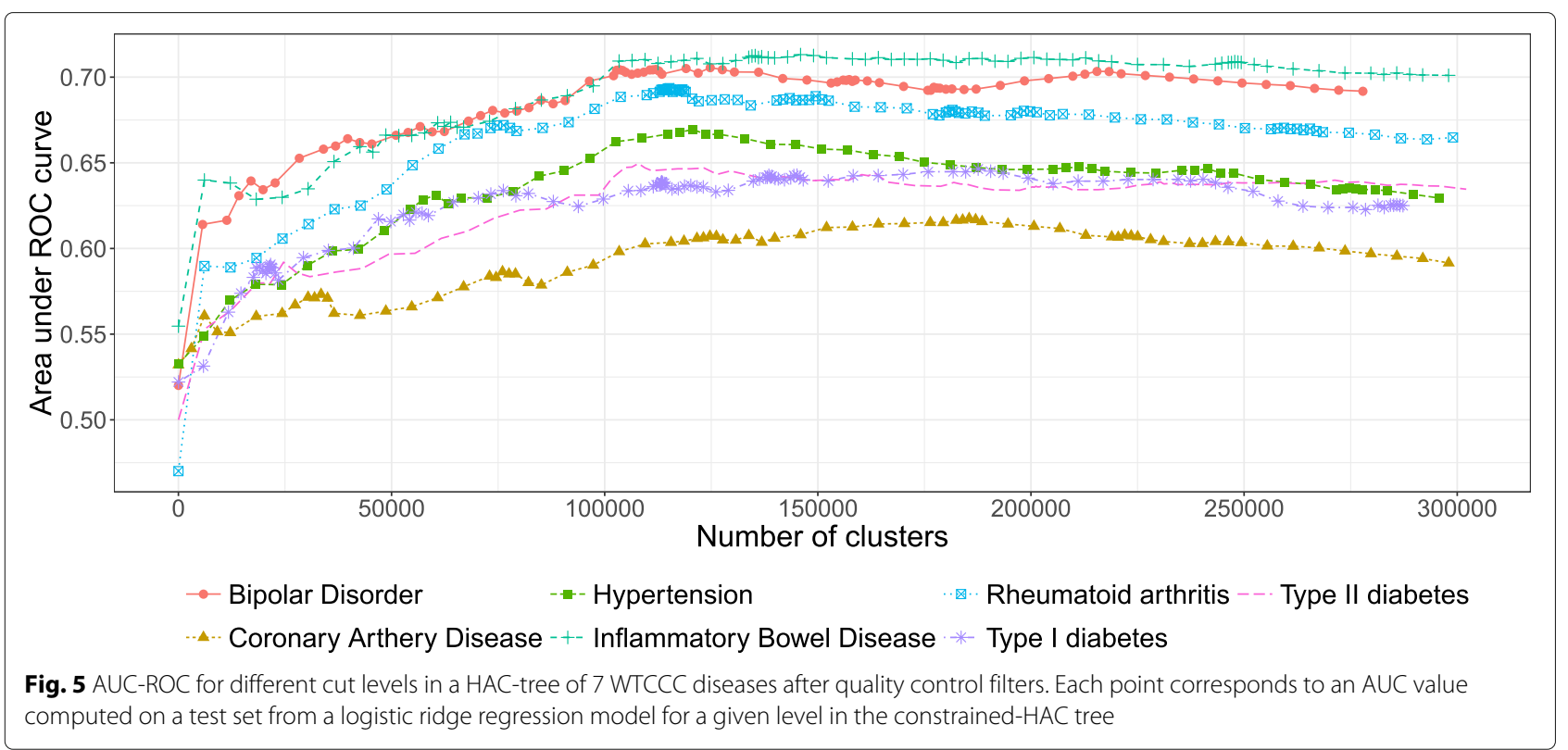




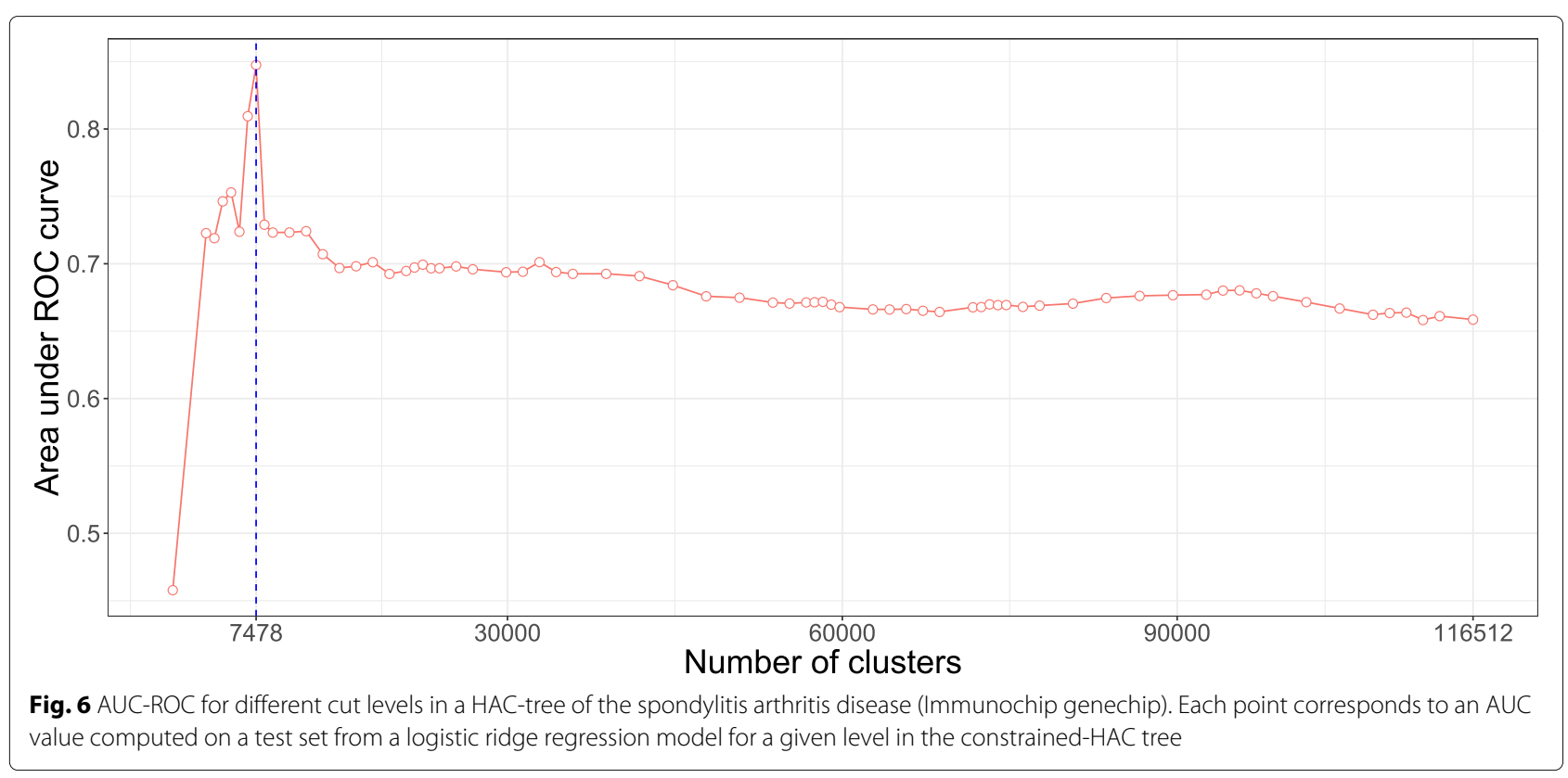

consists of the French subset of the large study of the International Genetics of Ankylosing Spondylitis (IGAS) study [22]. For this subset, unrelated cases were recruited through the Rheumatology clinic of Ambroise Paré Hospital (Boulogne-Billancourt, France) or through the national self-help patients' association: "Association Française des Spondylarthritiques". Populationmatched unrelated controls were obtained from the "Centre d'Etude du Polymorphisme Humain", or were recruited as healthy spouses of cases. The dataset contains 408 cases and 358 controls, and each individual was genotyped for 116,513 SNPs with Immunochip technology.

To remove the bias induced by population stratification in Genome-Wide analysis, we added the first 5 genomic principal components into the regression model as described in [37]. Since the methods evaluated here do not deal with missing values, we chose to impute the missing genotypes with the most frequent genotypic value, $h_{j}$ observed for each $j$ SNP.

For each each dataset, we filtered the values to keep only those SNPs having a MAF greater than $5 \%$. The

Table 1 Comparison of marker density and averaged LD level between markers in a region of 300 SNPs for the different datasets

\begin{tabular}{llll}
\hline Dataset & SNP/kb & Median & Mean \\
\hline Simulated data & $1.3 \times 10^{-27}$ & $1 \times 10^{-2}$ & 0.11 \\
WTCCC data & $7 \times 10^{-32}$ & $9 \times 10^{-4}$ & 0.03 \\
AS data & $9 \times 10^{-9}$ & $3 \times 10^{-2}$ & 0.27 \\
\hline
\end{tabular}

minor allele frequencies of each datasets are represented in Fig. 4.

We applied our cut level algorithm to find relevant clusters of SNPs and we performed single marker analysis on single SNPs (SMA) and on groups of SNPs (SASA, SKATtree, SKATnotree). We then compared the significant associations detected by the different methods to reveal possible new associations with the phenotype.

\section{Results in WTCCC and AS studies}

AUC-ROC curves In this section, we compare the AUCROC curves generated by our cut level algorithm for each disease (WTCCC and AS data).

Concerning the WTCCC diseases, given that patients were all genotyped using the same GeneChip, their genotypes have the same LD structure, and therefore the shapes of the AUC-ROC curves should be very similar between the different diseases. As can be observed in Fig. 5 (WTCCC diseases), the shape of the AUC-ROC curves are closely similar, with a chosen cut level located around 100000 clusters of SNPs, suggesting a shared LD pattern among patients.

In contrast, the AUC-ROC from the AS data (Fig. 6) behaves differently from the WTCCC data. Predictive power is substantially improved if aggregated-SNP predictors are used at a fairly high level in the hierarchical tree (7478 optimal clusters identified by the cut level algorithm). It is relevant to note that the pattern we observe on this real dataset is similar to the pattern we observed in the numerical simulations, especially under the clusSNP scenario. 
As we remarked concerning the WTCCC results, the algorithm identifies a relatively high number of clusters in relation to AS and simulated data. This difference is certainly due to the LD level among the genetic markers in the Affymetrix GeneChip. The correlation levels among SNPs for a given bandwith are similar between the simulated and the AS data, but greater than for the WTCCC data (Table 1 and Fig. 7). This suggests that there is a stronger LD pattern between blocks of SNPs in AS and simulated data, implying that the optimal number of clusters identified by the algorithm is dependent on the LD level among variables.

GWAS analysis on AS and WTCCC datasets To evaluate the ability of our procedure to discover new associations between SNPs and ankylosing spondylitis, we compare our procedure with the univariate approach (SMA) and SKAT model with our group definition and arbitrary group definition (20 SNPs). For SASA, we perform multiple hypothesis testing on the aggregated-SNP predictors in order to unravel significant associations with the phenotype. Fig. 8 presents the results of the association analysis. For each method the logarithm of the $p$-value of the different predictors is plotted along their position on the genome (this plot is also known as Manhattan plot).

Either methods highlight a region on chromosome 6 strongly associated with the phenotype. This region corresponds to the Major Histocompatibility Complex (MHC), and Human Leukocyte Antigen (HLA) class I molecules HLA B27 belonging to this region have been identified as a genetic risk factor associated with ankylosing spondylitis [38]. Our method SASA succeeds in detecting this risk locus with a good precision, 64 aggregated-SNPs variables are significantly associated with the phenotype compared to 602 significantly associated SNPs with the standard SMA approach.

For the analysis of the WTCCC datasets, we represent the results, in Fig. 9, by plotting the expected $p$-value against the observed $p$-value (this a plot is known as Quantile-Quantile plot). We perform the analysis using our approach SASA only.

\section{Discussion}

Overall,accounting for the linkage disequilibrium structure of the genome and aggregating highly-correlated SNPs is seen to be a powerful alternative to standard marker analysis in the context of GWAS. In terms of risk prediction, our algorithm proves to be very effective at classifying individuals given their genotype, while in terms of the identification of loci, it shows its ability to identify genomic regions associated with a disease with a higher precision than standard methods.

Is is also worth mentioning that our algorithm can also accomodate imputed variables as imputation in GWAS uses the Linkage Disequilibrium between variables to improve the coverage of variants. Our method being based on LD to define groups of common variants, we expect the group structure not to be impacted by imputation.

In this work we propose a four-step method explicitly designed to utilize the linkage disequilibrium in GWAS data. Our method combines, on the one hand, unsupervised learning methods that cluster correlatedSNPs, and on the other hand, supervised learning techniques that identify the optimal number of clusters and reduce the dimension of the predictor matrix. We evaluated the method on numerical simulations and real datasets and compared the results with standard singlemarker analysis and group-based approaches (SKATtree

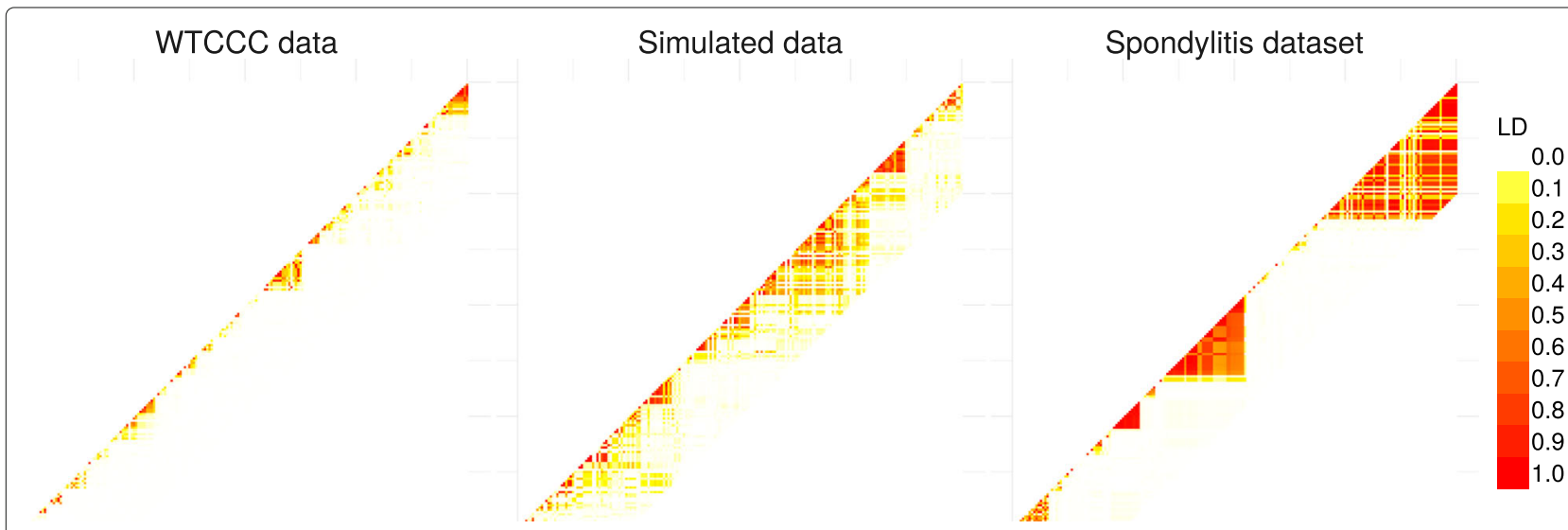

Fig. 7 Comparison of linkage disequilibrium level among SNPs for 3 different types of dataset: WTCCC, simulated and ankylosing spondylitis datasets. LD computation is based on $R^{2}$ between SNPs 


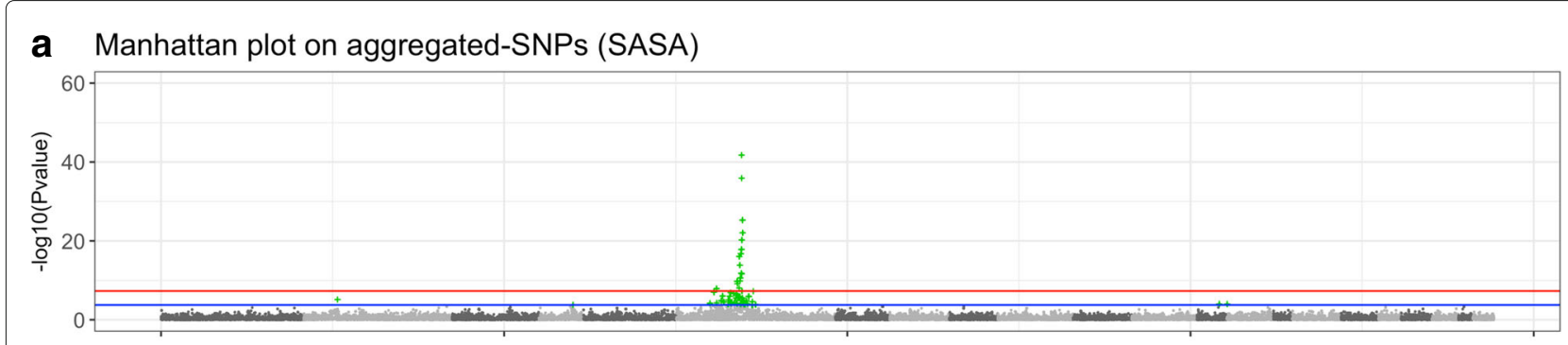

b Manhattan plot of single marker analysis

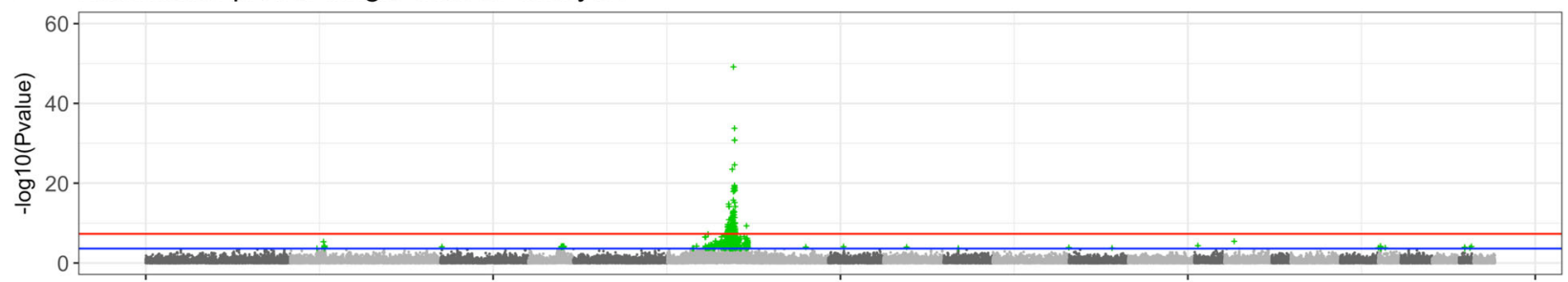

C Manhattan plot of SKAT analysis with arbitrary group definition

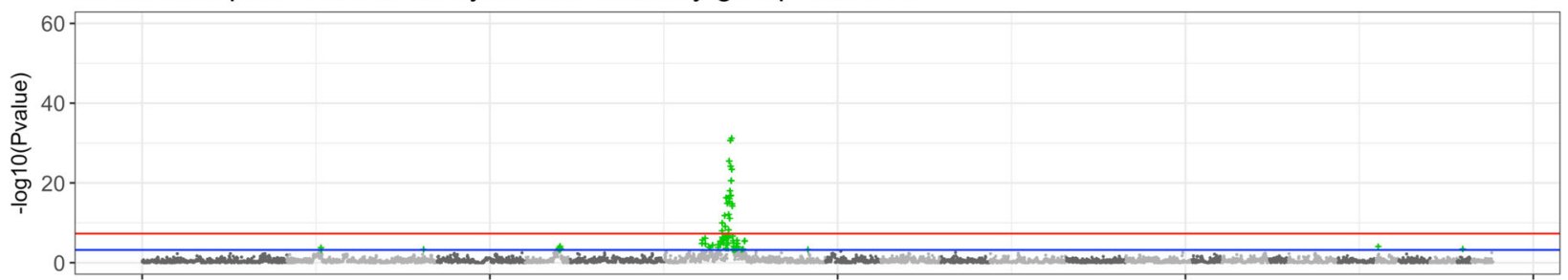

d Manhattan plot of SKAT analysis with tree-based group definition

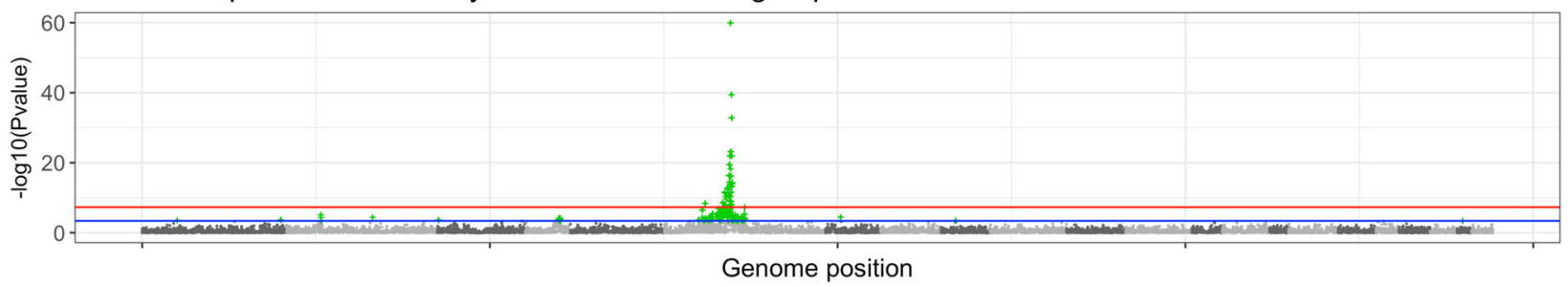

Fig. 8 Manhattan plots showing results of GWAS analysis on ankylosing spondylitis data. For each Manhattan plot, the Benjamini-Hochberg (BH) threshold is represented by the blue line and the Bonferroni threshold by the red line. According to the $\mathrm{BH}$ threshold, there are: (a) 64 significantly associated aggregated-SNPs; (b) 602 significantly associated single SNP; (c) 80 significantly associated groups of SNPs and (d) 138 significantly associated groups of SNPS

and SKATnotree). We remarked that the combination of our aggregating function with a ridge regression model leads to a major improvement in terms of predictive power when the linkage disequilibrium structure is strong enough, hence suggesting the existence of multivariate effects due to the combination of several SNPs. These results remained consistent across two applications involving several binary traits (WTCCC and ankylosing spondylitis datasets).

In terms of the identification of associated loci in different simulation scenarios, our method demonstrates its ability to retrieve true causal SNPs and/or clusters of SNPs with substantially higher precision coupled with a good power. On real GWAS data, our method has been able to recover a genomic region associated with ankylosing spondylitis (HLA region on chromosome 6) with a higher precision than standard single-marker analysis.

To improve our method further, while taking into account structured input variables in GWAS, there are different avenues that may be explored. One avenue would involve highlighting potential non-linear relationships between aggregated-SNPs and a response phenotype. This could be done by making use of the continuous nature of aggregated-SNPs variables (in contrast to the ordinal nature of single SNP variables), by using generalized additive models [39], and by performing non-linear regression 


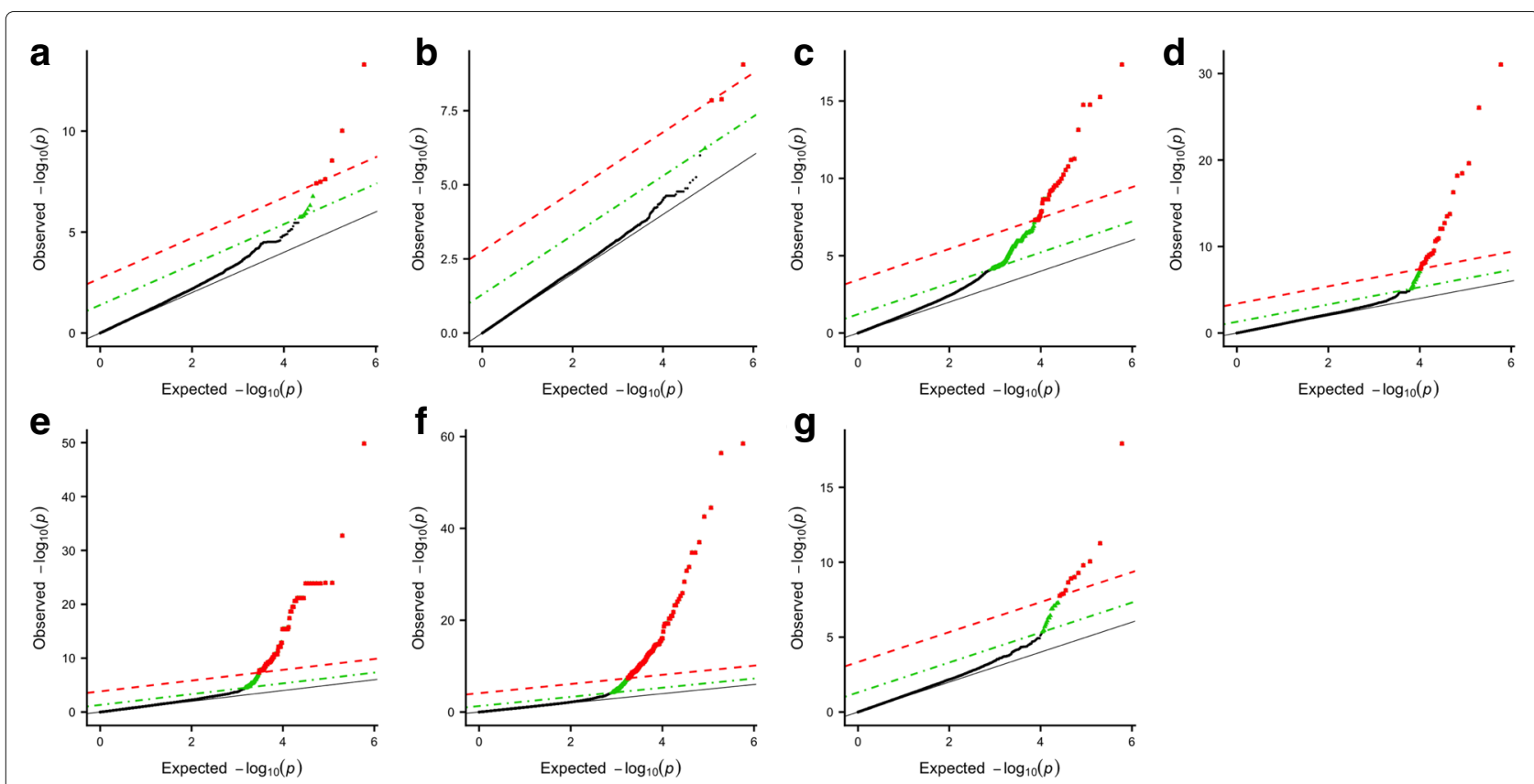

Fig. 9 Q-Q plots of group-based genome-wide analysis on WTCCC data using the SASA approach. For each Manhattan plot, the Benjamini-Hochberg $\mathrm{BH}$ ) threshold is represented by the green dotted line and the Bonferroni threshold by the red dashed line. (a) Bipolar disorder - 13 significant clusters of SNPs; (b) Coronary arthery disease - 4 significant clusters of SNPs; (c) Inflammatory bowel disease - 356 significant clusters of SNPs; (d) Hypertension - 47 significant clusters of SNPs; (e) Rheumatoid arthritis - 202 significant clusters of SNPs; (f) Type I diabetes - 358 significant clusters of SNPs; (g) Type II diabetes - 28 significant clusters of SNPS

using natural polynomial splines. In addition, whereas we evaluated our method for binary traits (case-control phenotype), a possible extension might include quantitative non-binary traits (i.e., using a ridge regression model instead of logistic ridge regression).

\section{Abbreviations}

FAIS: Fast automatized interval search GWAS: Genome-wide association studies HLA: Human leukocyte antigens LASSO: Least absolute shrinkage and selection operator LD: Linkage disequilibrium MAF: Marker allele frequency MHC: Major histocompatibility complex ROC: Receiver operating characteristic SASA: Single aggregated-SNP analysis SNP: Single sucleotide polymorphism SMA: Single marker analysis SKAT: Sequence kernel association test WTCCC: Wellcome trust case-control consortium

\section{Acknowledgements}

This study makes use of data generated by the Wellcome Trust Case-Control Consortium. A full list of the investigators who contributed to the generation of the data is available from www.wtccc.org.uk. Funding for the project was provided by the Wellcome Trust under award 076113, 085475 and 090355 .

\section{Funding}

UMR 8071 LaMME - UEVE, CNRS, ENSIIE, USC INRA, 23 bd de France, 91000

Evry, France. BIOptimize, 51000 Reims, France.

\section{Availability of data and materials}

A web server to run the proposed approach (coded in $\mathrm{R}$ ) is available at this adress: http://stat.genopole.cnrs.fr/leos.

\section{Authors' address}

Florent Guinot 1,2 : florent.guinot@genopole.cnrs.fr; Marie Szafranski : marie.szafranski@math.cnrs.fr; Christophe Amroise ${ }^{1,3}$ : christophe.ambroise@genopole.cnrs.fr; Franck Samson? : franck.samson@jouy.inra.fr

\section{Authors' contributions}

FG, CA and MS developed the approach and the design. FG conducted the simulation study, applied the approach to the real data and drafted the manuscript. MS and CA supervised the implementation process and critically read and edit the manuscript. FS developped the webserver and made it available online. The work of FG fulfills part of the requirements of his PhD. All authors read and approved the final manuscript.

\section{Ethics approval and consent to participate}

The dataset regarding ankylosing spondylitis consists of the French subset of the large study of the International Genetics of Ankylosing Spondylitis (IGAS) study [22]. For this subset, unrelated cases were recruited through the Rheumatology clinic of Ambroise Paré Hospital (Boulogne-Billancourt, France) or through the national self-help patients' association: "Association Française des Spondylarthritiques". Population-matched unrelated controls were obtained from the "Centre d'Etude du Polymorphisme Humain", or were recruited as healthy spouses of cases. The protocol was reviewed and approved by the Ethics committee of the Ambroise Paré hospital. All participants gave their informed consent to the study.

\section{Consent for publication}

Not applicable.

\section{Competing interests}

The authors declare that they have no competing interests.

\section{Publisher's Note}

Springer Nature remains neutral with regard to jurisdictional claims in published maps and institutional affiliations.

\section{Author details}

${ }^{1}$ UMR 8071 LaMME - UEVE, CNRS, ENSIIIE, USC INRA, 23 bd de France, 91000 Evry, France. ${ }^{2}$ BIOptimize, 51000 Reims, France. ${ }^{3}$ UMR MIA-Paris -

AgroParisTech, INRA, Université Paris-Saclay, 75005 Paris, France. 
Received: 29 September 2017 Accepted: 9 November 2018

Published online: 29 November 2018

\section{References}

1. Benjamini Y, Hochberg Y. Controlling the false discovery rate: A practical and powerful approach to multiple testing. J R Stat Soc Ser B. 1995;57(1): 289-300.

2. Dalmasso C, Broët P, Moreau T. A simple procedure for estimating the false discovery rate. Bioinformatics. 2005;21:660-8.

3. Maher B. Personal genomes: The case of the missing heritability. Nat News. 2008;456(7218):18-21.

4. Lee $S$, Abecasis GR, Boehnke M, Lin X. Rare-variant association analysis: study designs and statistical tests. Am J Hum Genet. 2014;95(1):5-23.

5. Manolio TA, Visscher PM. Finding the missing heritability of complex diseases. Nature. 2009;461(7265):747-53.

6. Asimit JL, Day-Williams AG, Morris AP, Zeggini E. ARIEL and AMELIA: testing for an accumulation of rare variants using next-generation sequencing data. Hum Hered. 2012;73(2):84-94.

7. Wu MC, Lee S, Cai T, Li Y, Boehnke M, Lin X. Rare-variant association testing for sequencing data with the sequence kernel association test. Am J Hum Genet. 2011;89(1):82-93.

8. Lee $\mathrm{S}, \mathrm{Wu} M C$, Lin X. Optimal tests for rare variant effects in sequencing association studies. Biostatistics. 2012;13(4):762-75.

9. Meinshausen N. Hierarchical testing of variable importance. Biometrika. 2008;95(2):265-78.

10. Holm S. A simple sequentially rejective multiple test procedure. Scand J Stat. 1979;6(2):65-70.

11. Ardlie $K G$, Kruglyak $L$, Seielstad M. Patterns of linkage disequilibrium in the human genome. Nat Rev Genet. 2002;3(4):299-309.

12. Gabriel SB, Schaffner SF, Nguyen H, Moore JM, Roy J, Blumenstiel B, Hig gins J, DeFelice M, Lochner A, Faggart M, L-C SN, Rotimi C, Adeyemo A, Cooper R, Ward R, Lander ES, Daly MJ, Altshuler D. The structure of haplotype blocks in the human genome. Science. 2002;296(5576):2225-9.

13. Huang BE, Amos Cl, Lin DY. Detecting haplotype effects in genomewide association studies. Genet Epidemiol. 2007;31(8):803-12.

14. Wu MC, Kraft P, Epstein MP, Taylor DM, Chanock SJ, Hunter DJ, Lin X. Powerful SNP-Set Analysis for Case-Control Genome-wide Association Studies. Am J Hum Genet. 2010;86(6):929-42.

15. Listgarten J, Lippert C, Kang EY, Xiang J, Kadie CM, Heckerman D. A powerful and efficient set test for genetic markers that handles confounders. Bioinformatics. 2013;29(12):1526-33.

16. Yoo YJ, Sun L, G Poirier J, D Paterson A, B Bull S. Multiple linear combination (MLC) regression tests for common variants adapted to linkage disequilibrium structure: Yoo et al. Genet Epidemiol. 2016;41. https://doi.org/10.1002/gepi.22024.

17. Paré G, Asma S, Deng WQ. Contribution of Large Region Joint Associations to Complex Traits Genetics. PLoS Genet. 2015;11. https://doi. org/10.1371/journal.pgen.1005103.

18. Kwak I-Y, Pan W. Adaptive gene- and pathway-trait association testing with GWAS summary statistics. Bioinformatics. 2016;32:1178-84. https:// doi.org/10.1093/bioinformatics/btv719.

19. Petersen A, Alvarez C, DeClaire S, Tintle NL. Assessing Methods for Assigning SNPs to Genes in Gene-Based Tests of Association Using Common Variants. PLOS ONE. 2013;8. https://doi.org/10.1371/journal. pone.0062161.

20. Purcell S, Neale B, Todd-Brown K, Thomas L, Ferreira MAR, Bender D Maller J, Sklar P, De B PIW, Daly MJ, et al. Plink: a tool set for whole-genome association and population-based linkage analyses. Am J Hum Genet. 2007:81(3):559-75.

21. WTCCC. Genome-wide association study of 14,000 cases of seven common diseases and 3,000 shared controls. Nature. 2007:447(7145): 661-78.

22. International Genetics of Ankylosing Spondylitis Consortium (IGAS), Cortes A, Hadler J, Pointon JP, Robinson PC, et al. Identification of multiple risk variants for ankylosing spondylitis through high-density genotyping of immune-related loci. Nat Genet. 2013;45(7):730-8.

23. Dehman A, Ambroise C, Neuvial P. Performance of a blockwise approach in variable selection using linkage disequilibrium information. BMC Bioinformatics. 2015;16:148.

24. Ward JH. Hierarchical Grouping to Optimize an Objective Function. J Am Stat Assoc. 1963;58(301):236-44
25. Tibshirani R, Walther $G$, Hastie T. Estimating the number of clusters in a data set via the gap statistic. J R Stat Soc Ser B. 2001;63(2):411-23.

26. Hartigan JA. In: Wiley Series in Probability and Mathematical Statistics, editor. Clustering algorithms: Wiley; 1975.

27. Caliński T, Harabasz J. A dendrite method for cluster analysis. Commun Stat. 1974;3(1):1-27

28. Krzanowski WJ, Lai YT. A criterion for determining the number of groups in a data set using sum-of-squares clustering. Biometrics. 1988;44(1): 23-34.

29. Bousquet O, Elisseeff A. Stability and Generalization. J Mach Learn Res. 2002;2:499-526.

30. Bonnet A, Gassiat E, Lévy-Leduc C. Heritability estimation in high dimensional linear mixed models. ArXiv e-prints. 2014. http://arxiv.org/ abs/1404.3397.

31. Nelder JA, Wedderburn RWM. Generalized Linear Models. J R Stat Soc: Ser A 1972;135(3):370-84

32. Sham PC, Purcell SM. Statistical power and significance testing in large-scale genetic studies. Nat Rev Genet. 2014;15(5):335-46.

33. Su Z, Marchini J, Donnelly P. Hapgen2: simulation of multiple disease snps. Bioinformatics. 2011;27(16):2304.

34. Diaz-Quijano FA. A simple method for estimating relative risk using logistic regression". BMC Med Res Methodol. 2012;1(1):14.

35. Brzyski D, Peterson CB, Sobczyk P, Candès EJ, Bogdan M, Sabatti C. Controlling the Rate of GWAS False Discoveries. Genetics. 2017;205: https://doi.org/10.1534/genetics.116.193987.

36. Yi H, Breheny P, Imam N, Liu Y, Hoeschele I. Penalized multimarker vs. single-marker regression methods for genome-wide association studies of quantitative traits. Genetics. 2015;199(1):205-22.

37. Price AL, Patterson NJ, Plenge RM, Weinblatt ME, Shadick NA, Reich D. Principal components analysis corrects for stratification in genome-wide association studies. Nat Genet. 2006;38:904-9.

38. Woodrow JC, Eastmond CJ. HLA B27 and the genetics of ankylosing spondylitis. Ann Rheum Dis. 1978;37(6):504-9.

39. Breiman L. Fitting additive models to regression data. diagnostics and alternative views. Comput Stat Data Anal. 1993;15:13-46.
Ready to submit your research? Choose BMC and benefit from:

- fast, convenient online submission

- thorough peer review by experienced researchers in your field

- rapid publication on acceptance

- support for research data, including large and complex data types

- gold Open Access which fosters wider collaboration and increased citations

- maximum visibility for your research: over 100M website views per year

At $\mathrm{BMC}$, research is always in progress.

Learn more biomedcentral.com/submissions 